Journal of Bangladesh Academy of Sciences, Vol. 36, No. 1, 53-59, 2012

\title{
EFFECTS OF INJURY BY A PYRALID INSECT PEST ON DUCKWEED GROWTH AND PRODUCTIVITY IN MINIPOND ECOSYSTEM
}

\author{
N. PARVEN*, M. A. BASHAR, M. A. K. CHOWDHURY AND S. B. QURAISHI ${ }^{1}$ \\ Environmental Biology and Biodiversity Laboratory, Department of Zoology, University \\ of Dhaka, Dhaka-1000, Bangladesh
}

\begin{abstract}
The strategic points of a pyralid insect, Synclita occidentalis (Pylralidae, Lepidoptera) on duckweed productivity, life cycle and its association with duckweed (host-plant) in pond ecosystem was studied. A total of 16 samples were used to study the infestation intensity and the impact of pest on duckweed production. The insect was found deeply associated with duckweed to complete its life cycle. The pyralid insect attains its pest status at larval stage and continues up-to the pupal stage. Larval and pupal case-making and feeding adaptations were noted as the characteristic nature of injury. The larvae build case with duckweed fronds and roots and use duckweed fronds that failed to multiply further. The crop of larva was the largest and widest part of the alimentary canal and varied exceedingly along with larval category. The experimental result indicated that pest impact in two different experimental miniponds was different in quantities but similar in mode of action.
\end{abstract}

Key words: Duckweed, Pyralid insect, Pyralid and duckweed interaction, Pond ecosystem

\section{INTRODUCTION}

Duckweeds are small floating aquatic plants (Journey et al. 2001). In Bangladesh, duckweed appears to be a suitable food for a wide range of fish species within monoculture and polyculture system (Gaingher et al. 1993, Hajra and Thipathi 1985). This weed contains a lot of minerals. The carbohydrate and protein amounts are noticeable, which are valuable food source (Landolt and Khandoker 1987). Duckweed is an aquatic vascular plant and proved to be a good accumulator of some toxic metals (Zayed 1998). A few farms in Bangladesh are trying to increase and establish the culture of duckweed to raise the important protein source of the country. The productions of duckweeds are highly hampered due to attack of a pyralid insect Synclita occidentalis that attains its pest status at the larval and pupal stages (Taleker et al. 1991, Pata and Ekbom 1992, Labatte and Got 1993). The larval feeding habit and case making adaptability of both the larva and pupa give the insect a pest status (Bashar and Aslam 1998). The larvae build case with the duckweed fronds and roots and the used duckweed fronds fail to multiply further

*Corresponding author: <parvennousheen@yahoo.com>.

${ }^{1}$ Chemistry Division, Atomic Energy Center, Dhaka-1000, Bangladesh. 
(Bashar and Aslam 1999). Immediately after hatching the larva begins to feed and starts causing damage to the host plant. The larval stage occupies greater part of its lifetime and consumes large amount of duckweed for its growth and development (Bashar et al. 2007). The host plant preference and infestation intensity increased by the availability of suitable leaf sheath for adult egg laying and larval development (Bashar et al. 2008). The present experiment emphasized to identify the life cycle pattern of this pyralid and the stage which causes maximum damage. Considering the great importance of duckweed, the objective of this study was to utilize the plant- insect association for the management of bio-resources in aquatic ecosystem.

\section{MATERIALS AND METHODS}

The duckweed bio-mass (both infested and non-infested units), the pyralid insect eggs with duckweed, larval cases with larvae, pupal cases with pupae and adults were collected from two different minipond-ecosystem of Mirzapur Kumudini Hospital Complex (Tangail). These were organic and inorganic ponds. The organic pond was supplied with organic matters (drainage effluent like domestic, municipal and hospital wastes) and the inorganic pond was with inorganic materials (cow-dung, decomposed materials and inorganic fertilizers) as duckweed nutrients. A total 16 samples were collected (June, 2006 to August, 2006) at seven days of intervals from the selected sites of the selected ponds by metallic sieve $(6.35 \mathrm{~cm} \times 6.35 \mathrm{~cm})$ and stored in $(11.43 \mathrm{~cm} \times$ $8.89 \mathrm{~cm}$ ) plastic containers. Samples were kept in a pot containing tap water covered with mosquito net. Samples were placed near the windows to ensure constant supply of air.

The life cycle study was conducted in the experimental beakers $(11.43 \mathrm{~cm} \times 8.89$ $\mathrm{cm})$ with duckweed biomass and pond water. Different larval categories were measured in millimeter. More duckweed fronds were supplied for compensating the food shortage in the experimental containers. The egg laying behavior, incubation period, larval and pupal period were recorded at 24 hours intervals following the methods described by Elora et al. (2002) and Bashar et al. (2007).

Date-wise collected duckweed infested fronds number were recorded. Larval abundance, number of pupae and adults were also recorded by visual observations to find out the infestation intensity following the methods of Bashar and Aslam (1999). Selected duckweed infested units were observed under SKT binocular microscope to estimate the infestation intensity according to the methods of Bashar et al. (2008). Mode of infestation and infestation intensity due to larval feeding were measured by following Israfil and Abraham (1967). 
Total 152 larval and 15 pupal cases were collected and examined the case-making adaptability and impact on duckweed productivity by following the methods of Bashar et al. (2007). Full fed larvae (32) were dissected in normal saline water $(\mathrm{NaCl} 0.9 \%)$ to examine the adaptations of feeding habit. Different parts of the digestive tract was measured with an occulometer calibrated against a stage micrometer following the methods of Bashar et al. (2008).

\section{RESULTS AND DISCUSSION}

Developmental stages of pyralid insect directly depend on the host plant (duckweed) for food and shelter. The association with duckweed at adult stage, incubation period, larval period and pupal stage was 12,16, 64 and 24\%, respectively. Twenty hours after mating, the female started to oviposite eggs in cluster on the under surface of duckweed fronds. The incubation period was found to vary 3 - 4 days. Merrit and Cummins (1995) found that the incubation periods of aquatic pyralid insect range from 6 - 11 days. Larval period was found to vary from 16 to18 days. Except the size and colour variation very little morphological differences were observed.

Total larval abundance of inorganic pond sample was $61 \%$ higher than organic $39 \%$ during the study period (Fig. 1). The larvae feed on duckweed fronds and used fronds failed to multiply further and infestation took place (Figs 6 and 7). The infestation intensity and duckweed biomass varied along with the larval abundance as was found by Bashar and Aslam(1999). The pyralid larva consumed large amount of food for its growth and development as was reported by Bashar et al. (2008).

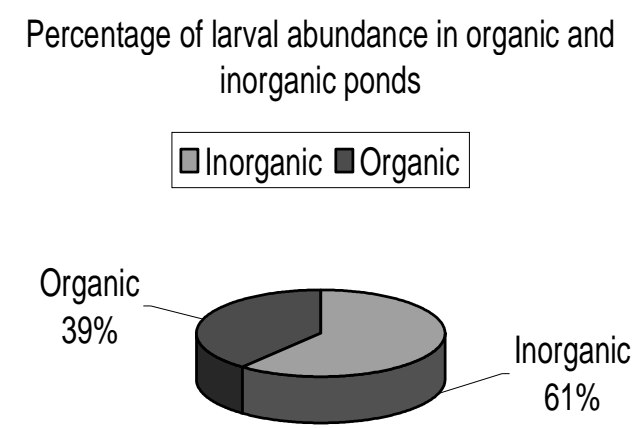

Fig. 1. Total larval abundance during the study period of inorganic samples.

The infested duckweed units of organic and inorganic pond samples varied greatly due to higher larval abundance in inorganic pond than in organic pond. The highest number of infested units of inorganic pond was 56 and that of organic was 31 among the 
sample units (Fig. 2). The number of infested fronds was also directly related with the number of roots (Fig. 1). The larval feeding behavior, abundance and larval durations were important factors which considered for the question of pest impact on duckweed production.

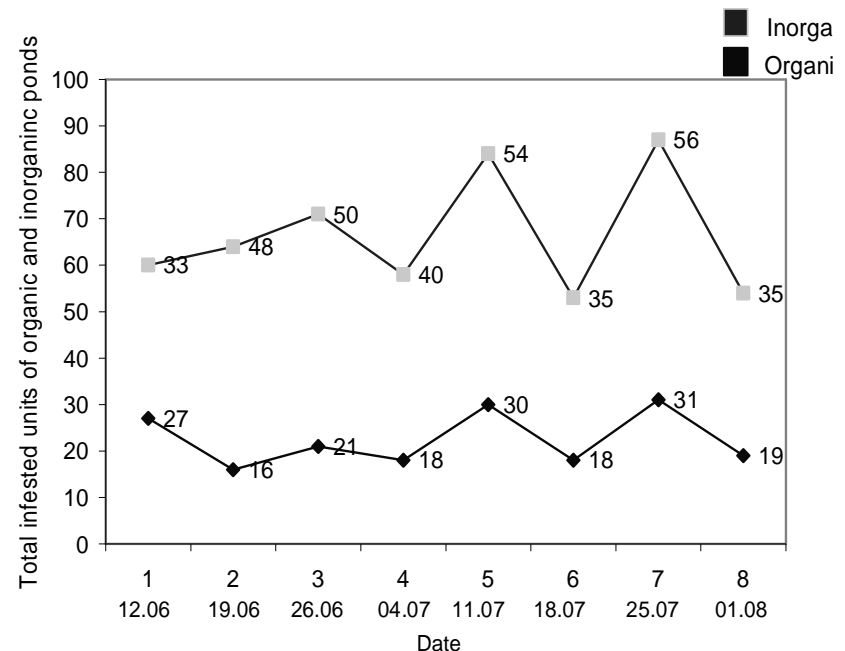

Fig. 2. Infested duckweed fronds number in organic and inorganic pond; infestation intensity was higher in inorganic pond than organic.

Roots of the infested units were broken and became brownish. The root cups were absent in most of the infested roots. Among two experimental ponds, in the inorganic pond the insect appeared as a serious pest causing significant damage to duckweed roots. The percentage of the number of roots in the sampled duckweed units of inorganic pond was 43 and in case of organic it was 57 (Fig. 3). The insect association with host plant was evidenced on roots damage by feeding activities. About $63 \%$ of duckweed fronds became rootless as reported by Bashar and Aslam (1998).

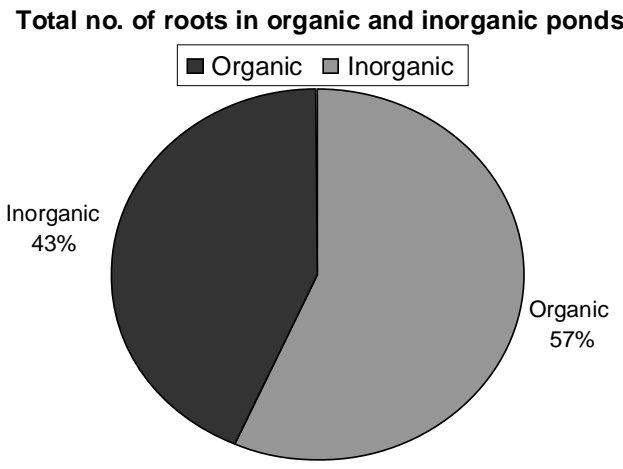

Fig 3. Total root percentage of collected duckweed units of organic and inorganic samples. 
Among 152 larval cases, 64\% were found to be made of 5 - 9 fronds and about $1.31 \%$ cases were with 15 - 19 fronds (Fig. 4). The term 'root damage' indicated the number of used roots which has been eaten up by larva or broken during construction of larval cases. It was recorded that more than $80.92 \%$ cases were with 0 - 2 roots. Most of the cases were without roots and defined as state 1 , only $1.31 \%$ cases were $9-10$ roots of duckweed fronds and defined as state 3 (Fig. 5).

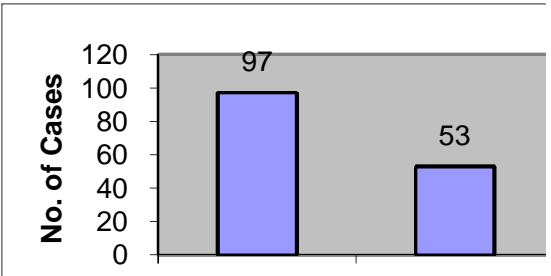

Fig. 4. Ranges of duckweed fronds in larval case construction.

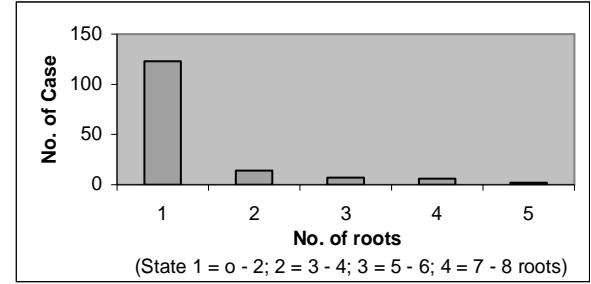

Fig. 5. Ranges of duckweed roots used in larval case construction.

The larval case-making is itself a special type of adaptability for the insect. The adaptability for larval stage lies in mechanism of case-making strategy; and it causes the duckweed frond-damage. The case is waterproof internally and contains a pocket of air. The inner layer of the case makes a close association with the source of oxygen within duckweeds (Fig. 7). According to Bashar and Aslam (1998) the pyralid larval casemaking adaptability is special for the aquatic mode of life. It is difficult to control this insect at the larval stage due to the presence of protective larval case (Akiko et al. 2002).

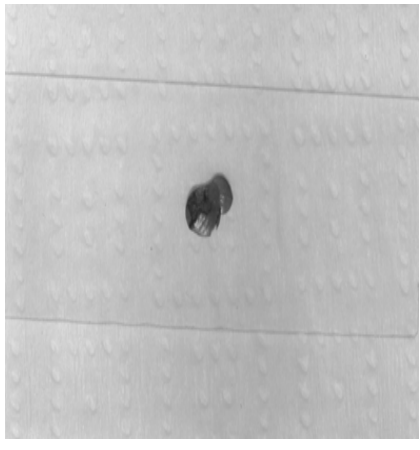

Fig. 6. Infested duckweed unit.

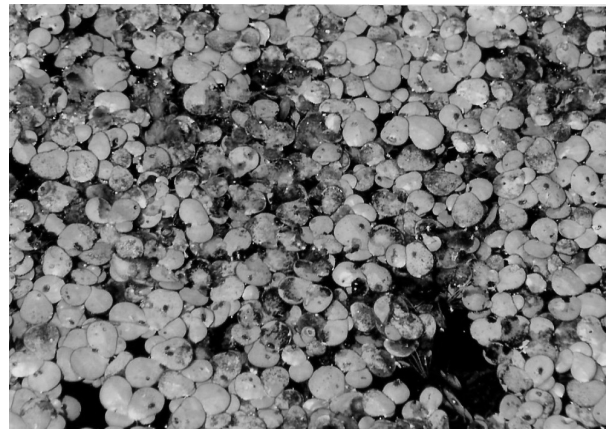

Fig. 7. Infested duckweed biomass by pyralid insect infestation.

The case-making adaptability at the pre-pupal stage found highly significant. Some new fresh duckweed fronds were attached with last larval case. The internal white color gelatinous sheath of larval cases becomes rigid and strong at this stage (Fig. 8). The pupal case was characteristically triactinal in appearance. 
The alimentary canal of larva was found highly adapted to consume duckweed fronds and roots. The maximum and minimum length and width of digestive tract were 6 and $3 \mathrm{~mm}$, respectively. Crop was the largest part of the alimentary canal and remained filled with the duckweed fronds and root materials, it occupied about $66.9 \%$ of the alimentary canal.
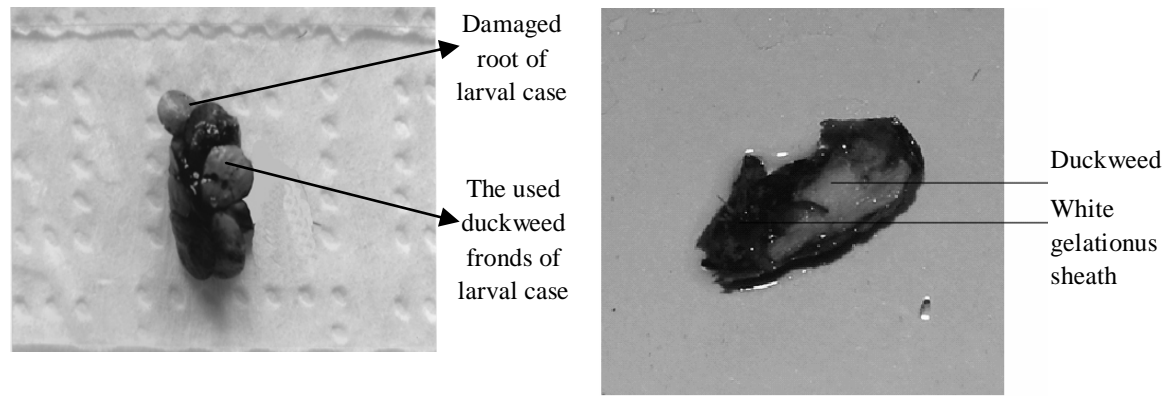

Fig. 8. Larval case-making adaptability in aquatic ecosystem (8X).

Fig. 9. Internal phase of larval case (2.5X).

The total amount of fat bodies were directly correlated with the amount of food (duckweed fronds) present in the dissected larvae. The relationship between the amount of fat bodies and food fronds showed significant value at $0.011 \%$ levels. The entire gastric caeca was a sac-like structure with distinct duct, measuring $9-20 \mathrm{~mm}$ in length and $0.50 \mathrm{~mm}$ in widths. Secretion of gastric caeca played an important role in digestion and excretion of duckweed fronds (Basher et al. 2008).

\section{CONCLUSION}

The herbivore pyralid had both direct and indirect interactions with host plant duckweed that negatively affected all aspects of plant growth including reproduction and status in a community. The data presented in this paper provided information on the insects association and interaction with its host plant (duckweed) in aquatic ecosystem and showed that the adaptability of this insect at larval stage was very distinct in morphological, physiological and behavioral aspects. The adaptability achieved by larval case-making exhibited a strong interaction of pyralid insect with duckweed in aquatic ecosystem. The adaptability strengthens the insect to have strategy of established plantanimal association in aquatic ecosystem.

\section{REFERENCES}

Akiko, F., M. Murakami, K. Kotaro, N. Mandatoshi and O.Takayki. 2002. A leaf-roller caterpillar improves the leaf quality. Biochem. Sci. 5: 263-266.

Bashar, M.A., N. Parven and M.A.K. Chwdhury. 2008. A case-making insect pest (Synclita occidentalis : Pyralidae) and its characteristic association with duckweed in minipond ecosystem. Dhaka Univ. J. Biol. Sci. 17: 109. 
Bashar, M. A. and A.F.M. Aslam. 1998. An evolution of the pest status of a pyralid insect (Synclita occidentalis) affecting duckweed productivity in a minipond ecosystem. Basngladesh J. Zool. 26: 35 .

Bashar, M. A. and A.F.M. Aslam. 1999. A pyralid pest on duckweed in minipond ecosystem and its characteristics nature of injury. Bangladesh J. Life Sci. 11: 117.

Bashar, M.A., N. Parven, and S.B. Quraishi. 2007. Larval adaptations of a pyralid insect pest (Synclita occidentalis) to its host-plant in pond ecosystem. Banglasesh J. Zool. 35: 366

Elora B. K., N.D. Islam and Z. Ferdousi. 2002. Some biological investigations and damage of vegetable crops by Epilachna pusillanima (Coleoptera:Coccinellidae). Rajshashi. Univ. J. Zool. 22: 77-78.

Gaingher, I. G., D. Prothab and G. Granoth. 1993. Evalution of duckweed (Limna gilbba) as feed for tilapia (Oreochromis niloticus) in a re-circular unit. Aquaculture 41: 235.

Hajra, A. and S.D. Thipathi. 1985. Nutritive value of aquatic weed Spirodella polyrhiza (Linn) in grass carp. Indian J. Aniam. Sci. 55: 702

Israfil, P. and T. P. Abrahim. 1967. In: Major insect pests of rice. John Hopkins Press, Baltimore, pp. 265-274.

Journey,W. K., P. Skillicorn and W. Spira . 2001. Duckweed Aquaculture: A new aquatic farming system for Developing countries. Technical department, Agriculture division, The World Bank. pp.11-15.

Labatte, J.M. and B. Got. 1993. Modeling within plant distribution of Europian Corn borer (Lepidoptera: Pyralidae) larvae on corn for infestation. J. Econ. Entomol. 83: 747-760.

Landolt, E. and R. Khandoker. 1987. Biosystematics investigations in the family of Lemnanaceae a monographic study. Veroffenlichugen des Geobatanisches Institutes der ETH, Stiffing Rubel, Zurich. Helf. pp.110.

Merrit, R.W. and K.W.Cummins. 1995. An introduction to the aquatic insects of North America. Third edition. Kendall publishing company, 4050, West Mark Drive Dubugue, Iowa 52002.

Pata, P. and B. Ekbom. 1992. Infestation and dispersal of early instars of Chilo partellus(Lepidoptera: Pyralidae) at different densities. Environ. Entomol. 21: 110.

Talekar, N. S., C.P. Lin, M.Y. Yin, Y.D. Wang and D.C.Y. Chang 1991. Characteristic nature of infestation by Ostrinia furnacalis (Lepidoptera:Pyralidae) in mungbean. J. Econ. Entomol. 85: 149 .

Zayed, A.1998. Phyto-accumulation of trace elements by wetland plant duckweed. J. Environ. Qual. 27: 715 . 